\title{
Parámetros genéticos para características de crecimiento, canal, calidad y espinas intramusculares en cachama blanca (Piaractus brachypomus)
}

\section{Genetic parameters for growth, carcass, quality and intramuscular spine traits in white blanca (Piaractus brachypomus)}

\author{
Gerald F. Bernal-Buitrago ${ }^{\text {; }}$ Jeferson A. Valderrama²; Daniela Monroy-Suárez³ \\ Carlos Manrique-Perdomo ${ }^{4}$; Víctor M. Medina-Robles ${ }^{5}$
}

\begin{abstract}
'Zootecnista. M.Sc., Grupo de Investigación sobre Reproducción y Toxicología de Organismos Acuáticos - GRITOX, Instituto de Acuicultura - IALL. Universidad de los Llanos, Villavicencio, Meta, Colombia, e-mail: gebernal@udca.edu.co, Dhttp://orcid.org/0000-0003-2013-8464

ZZootecnista, Grupo de Investigación sobre Reproducción y Toxicología de Organismos Acuáticos - GRITOX. Universidad de los Llanos, e-mail: jvalderrama@ unillanos.edu.co, Dhttp://orcid.org/0000-0001-5212-0889

${ }^{3}$ Estudiante de Licenciatura en Producción Agropecuaria. Universidad de los Llanos, e-mail: lizet.monroy@unillanos.edu.co, Dhttp://orcid.org/0000-00032609-698X

${ }^{4}$ Zootecnista, Ph.D., Facultad de Medicina Veterinaria y de Zootecnia. Universidad Nacional de Colombia, Bogotá, Colombia, e-mail: cmanriquep@unal.edu. co, (Dhttp://orcid.org/0000-0002-5824-6383
\end{abstract}

${ }^{5}$ MVZ, M.Sc, Grupo de Investigación sobre Reproducción y Toxicología de Organismos Acuáticos - GRITOX. Universidad de los Llanos, e-mail: vmmedinarobles@unillanos.edu.co, Dhttp://orcid.org/0000-0002-4871-2715

Cómo citar: Bernal-Buitrago, G.F.; Valderrama, J.A.; Monroy-Suárez, D.; Manrique-Perdomo, C.; Medina-Robles, V.M. 2019. Parámetros genéticos para características de crecimiento, canal, calidad y espinas intramusculares en cachama blanca (Piaractus brachypomus). Rev. U.D.C.A Act. \& Div. Cient. 22(1):e1182. https://doi.org/10.31910/rudca.v22.n1.2019.1182

Artículo de acceso abierto publicado por Revista U.D.C.A Actualidad \& Divulgación Científica bajo una licencia Creative Commons CC BY-NC 4.0

Recibido: Diciembre 7 de 2017

Aceptado: Febrero 21 de 2019

\section{RESUMEN}

Los temas de la acuicultura en los que se requiere innovación e investigación están orientados al mejoramiento y al conocimiento genético de especies nativas. En este sentido, el presente estudio se concentró en estimar heredabilidades, correlaciones genéticas y fenotípicas para características de crecimiento, canal, calidad y espinas intramusculares derechas (EIMD), en cachama blanca (Piaractus brachypomus). La progenie de 12 hembras y 24 machos por fertilización artificial de una hembra por dos machos, es decir, doce familias de hermanos medios, fueron analizados por medio del paquete estadístico SAS 9.4 (SAS $\left.{ }^{\circledR}, 2014\right)$. Se asumió un modelo lineal general univariado, con inclusión de los efectos fijos, como factores de entorno, estanque y edad y factores genéticos, como efectos aleatorios. Las heredabilidades, en general, para las variables de crecimiento, mostraron valores de medios a altos; para las variables de la canal presentaron valores bajos a medios; para las variables de calidad fueron bajos y, de particular interés para la EIMD, la magnitud de la estima fue de $0,11 \pm 0,15$. Las correlaciones estuvieron, generalmente, cercanas a la unidad entre características 
de crecimiento. Estas fueron significativamente positivas para peso corporal, a 180 días de edad, con la mayoría de variables, a diferencia con las variables de calidad, la correlación para rendimiento en canal con EIMD de -0,99 fue encontrada significativamente negativa. Los resultados del estudio destacan el potencial para mejorar características de crecimiento, de canal y de calidad, a través de la explotación de la variación genética aditiva observada.

Palabras clave: heredabilidades; cachama blanca; espina intramuscular; rendimiento en filete; mejoramiento animal.

\section{ABSTRACT}

The topics of aquaculture in which innovation and research are required are directed towards the improvement and genetic knowledge of native species. In this sense the present study concentrated on the estimation of heritabilities, genetic and phenotypic correlations for growth, carcass, quality and right intramuscular spines (RIMS) in white cachama (Piaractus brachypomus). The progeny of 12 females and 24 males by artificial fertilization of a female by two males, that is to say twelve half-sib families, were analyzed by means of the statistical package SAS $9.4\left(\right.$ SAS $\left.^{\circledR}, 2014\right)$, assumed an univariate general linear model with inclusion of Fixed effects such as environmental factors, pond and age as fixed effects and genetic factors as random effects. Heritabilities in general for growth variables showed medium to high values; for carcass variables, presented low to medium values; And for the quality variables were low; For the RIMS the magnitude of the estimate was $0,11 \pm 0,15$. The correlations were generally close to unity between growth characteristics. These were significantly positive for body weight at 180 days of age with most variables unlike quality variables; the correlation for channel performance with RIMS of -0.99 was found to be significantly negative. The results of the study highlight the potential to improve growth, carcass and quality traits through the exploitation of the additive genetic variation observed in this study.

Keywords: heritabilities; white cachama; intramuscular spine; fillet yield; animal breeding.

\section{INTRODUCCIÓN}

Según FAO (2016), la piscicultura continúa siendo la esfera de producción de alimentos de origen animal de más rápido crecimiento, siendo la alternativa más viable para cubrir la demanda y subsanar la seguridad alimentaria en los próximos años, a través del aumento en la eficiencia productiva, lo que implica mejorar las condiciones de manejo, infraestructura y calidad genética de las especies. En este contexto, la AUNAP et al. (2014) enfatizan que el reto de la cadena piscícola colombiana es involucrar los pilares fundamentales de una manera más global e integral, donde se le dé mayor importancia al mejoramiento genético de las especies nativas, con el propósito de transformar al sector en uno tecnificado y líder de la economía nacional, capaz de trascender al mercado internacional.

La cachama blanca (Piaractus brachypomus) es la segunda especie más comercial de aguas continentales colombianas; aun así, Mesa-Granda
\& Botero-Aguirre (2007) enfatizan que un desarrollo sostenible de esta especie en Colombia, además de optimizar los sistemas de producción, está ceñido al conocimiento genético de la especie, a un programa de mejora genética, debiendo centrarse en estimar componentes genéticos, tales como heredabilidades, correlaciones genéticas y fenotípicas entre caracteres productivos de mayor importancia.

Gjedrem et al. (2012) resaltan que, en todo programa de mejora genética, se tiene como finalidad manipular la frecuencia génica de los caracteres importantes de producción, teniendo en cuenta que, la mayoría, son cuantitativos y que muestran una variación hereditaria poblacional, debida a la segregación de varios genes, cuya expresión puede ser modificada por acción del medio, conllevando a que la relación entre fenotipo y genotipo no sea perfecta, es decir, como dos individuos con genotipos idénticos pueden tener fenotipos distintos; asimismo, la igualdad fenotípica no implica igualdad genética. Por dichas razones, según Bernal \& Gallego (2016) es indispensable estimar, por medio de la heredabilidad $\left(h^{2}\right)$, en qué medida la varianza fenotípica corresponde a la varianza, debida a los genes, de qué forma se relacionan los caracteres por medio de correlaciones genéticas $\left(\mathrm{r}_{\mathrm{g}}\right)$ y su evolución respecto con la edad de los animales. Dichos componentes son parámetros genéticos que, basados en medidas familiares o fenotípicas individuales, determinan la respuesta a la selección, establecen la estrategia a ser usada en el mejoramiento de los caracteres y son esenciales para la construcción exitosa de decisiones en la selección y programas de mejoramiento genético.

En ese orden de ideas, el objetivo principal de esta investigación se basó en estimar heredabilidades y correlaciones genéticas de características de crecimiento, canal, calidad y EIMD en cachama blanca, bajo condiciones de cultivo comercial, buscando emprender un programa de cría selectiva, con criterios de decisión sobre el método de mejoramiento, aumentar sus tasas de crecimiento y disminuir el número de espina intramuscular, mejorando características de la canal y calidad.

\section{MATERIAL Y MÉTODOS}

La población experimental fue establecida en cinco explotaciones del sector privado, distribuidas en los municipios de Guamal, Acacías, Restrepo y Cumaral, del Departamento del Meta, Colombia. Los parentales conformados por 12 hembras y 24 machos de cachama blanca fueron la base para obtener 12 familias de hermanos medios. Se utilizó un agrupamiento anidado descrito por Cardellino \& Rovira (1987), donde el desove de una hembra fue fertilizado por dos machos independientes y excluyentes. La reproducción dirigida fue por inducción hormonal con extracto de hipófisis de carpa, estrujamiento en seco y siguiendo el protocolo descrito por Atencio (2001). Para disminuir los efectos ambientales temporales o permanentes de los parentales que, generalmente, se incluyen en la estimación de parámetros genéticos, se consideró disminuir dichos efectos del error en el modelo, por lo cual, se reprodujeron animales capturados del medio, que tenían edades similares e igual cantidad de desoves. Por otra parte, en las progenies no se consideró diferencia atribuible al sexo por no existir dimorfismo en la especie; además, los desoves 
fueron acordes con otros reportados, asegurando estimaciones en condiciones normales de producción.

Las familias obtenidas fueron tratadas en cada una de las fincas separadamente, bajo condiciones comerciales, desde su incubación hasta su posterior traslado a hapas, en tela de $3 \times 3 \times 0,8 \mathrm{~m}$, establecidas dentro de estanques en tierra, a una densidad de sesenta larvas por $\mathrm{m}^{2}$ hasta alcanzar la talla de $3 \mathrm{~cm}$. Posteriormente fueron llevadas a la Estación Piscícola del Instituto de Acuicultura - Iall, de la Universidad de los Llanos - Unillanos, ubicada a $12 \mathrm{~km}$ de la ciudad de Villavicencio, Meta, donde se adecuaron tres estanques en tierra de $40 \times 10 \mathrm{~m}$, divididos en cuatro secciones de $10 \times 10 \mathrm{~m}$ cada uno, para distribuir, aleatoriamente, las 12 familias por separado. Esto se realizó, con el fin de evaluar el efecto del estanque, eliminar el efecto de ambiente común del modelo y dar condiciones similares de manejo y ambiente a todas las familias.

El estudio, se llevó a cabo con un total de 1.800 animales, 150 por familia, a una densidad de dos animales por $\mathrm{m}^{2}$, hasta alcanzar la edad de 180 días post-eclosión. Durante toda la fase de cultivo, se registró semanalmente la temperatura y las condiciones fisicoquímicas del agua, tales como oxígeno disuelto, amonio, $\mathrm{pH}$, alcalinidad y conductividad y, en lo concerniente a la alimentación con respecto a su biomasa del 10 al 2\%, fue con concentrado comercial, a una frecuencia de dos veces por día, 6 días a la semana.

Las características de crecimiento, se evaluaron mensualmente durante el tiempo de cultivo; los peces engordados fueron sacrificados y, después, se tomaron las medidas de las características de canal; para la ejecución de estos procedimientos, se tomaron, aleatoriamente, veinte individuos por familia, atrapados en redes, posteriormente anestesiados, usando una solución de 2-fenoxietanol a $300 \mathrm{ppm}$ y, por último, fueron medidos, pesados, sacrificados y fileteados manualmente.

Las características de crecimiento evaluadas fueron: peso; longitud estándar (LE), medida en línea recta, desde el extremo distal de la mandíbula inferior hasta el límite posterior de la última vertebra; longitud total (LT), medida en línea recta, desde el extremo distal de la mandíbula inferior hasta el límite posterior de la aleta caudal; ancho del cuerpo (ACPO), medida desde el inicio de la aleta dorsal hasta la parte más distal de la quilla ventral. Las características de la canal evaluadas fueron: peso de la canal (PC), como peso del pez, una vez eviscerado manualmente; rendimiento en canal (RC), como peso de la canal, expresada en porcentaje del peso del pez sin eviscerar (100 x peso canal/peso pez); rendimiento en filete (RF), como peso del filete, expresado en porcentaje del peso del pez sin eviscerar (100 x peso filete/peso pez); espina intramuscular derecha (EIMD), donde se utilizaron los filetes derechos de cada animal, los cuales, se almacenaron en bolsas plásticas estériles, a $-20^{\circ} \mathrm{C}$, desde el momento del sacrificio hasta su posterior cuantificación, realizada de manera manual post-cocción.
Para evaluar las características de calidad, se pesaron los filetes izquierdos de la muestra de 20 peces, se almacenaron en bolsas plásticas estériles a $-20^{\circ} \mathrm{C}$, hasta el momento de la realización del análisis de la composición de la carne. En todos los casos, el análisis se efectuó por duplicado, tomándose la media como resultado definitivo. Humedad (HD), para esto, se molió una muestra del filete, posteriormente se introdujo en un crisol y se secó en la estufa, a $105^{\circ} \mathrm{C}$, durante 24 horas; Cenizas, las muestras se incineraron a $600^{\circ} \mathrm{C}$ en mufla, durante 3 horas, para destruir el material orgánico, dejando solo el material mineral; Grasa, a partir de otra muestra molida del filete, envuelta en un papel absorbente, se determinó la cantidad de grasa, mediante el método Soxhlet y Proteína (P), la determinación de nitrógeno total, se realizó mediante el método Microjkeldalh.

Para el análisis estadístico de los datos obtenidos durante el desarrollo del estudio y la estimación de los parámetros genéticos, se utilizó el paquete estadístico SAS 9.4 (SAS $\left.{ }^{\circledR}, 2014\right)$; la homogeneidad de la varianza fue comprobada mediante el estadístico Levene's F-test; la normalidad por la prueba de Kolmogorov-Smirnov con el procedimiento UNIVARIATE, que se usó también para calcular los estadísticos descriptivos de las variables y también para comprobar el efecto del estanque en todos los casos, mediante un ANOVA. Para estimar los componentes de varianza y covarianza, se utilizó el método de máxima verosimilitud restringida (REML), donde se asumió un modelo mixto univariado, con inclusión de los efectos fijos ambientales, como edad, estanque, condiciones de manejo y el efecto genético (familia), como factor aleatorio, así: $\mathrm{Y}=\mathrm{X} \beta+\mathrm{Zu}$ $+\mathrm{e}$, donde, $\mathrm{Y}$ es el dato registrado para la característica de estudio, $\beta$ y u vectores de efectos fijos y aleatorios respectivamente, $X$ y $Z$ son matrices de incidencia que relacionan las observaciones a los efectos fijados y aleatorios, respectivamente.

Las magnitudes de la estimación de la heredabilidad fueron interpretadas siguiendo la clasificación de Campos (2004), como: baja $0,05-0,19$; media 0,20 - 0,44; alta $0,45-0,64$ y muy alta $>0,65$. La correlación fue clasificada como: baja $0,0-0,44$, media $0,45-0,59$ y alta $0,60-1,0$. Las heredabilidades y correlaciones, junto con sus precisiones (error estándar), fueron estimadas siguiendo la metodología de agrupamiento de medio hermanos, lo que permitió también estimar solo las varianzas aditivas y ,por lo tanto, estimaciones insesgadas de los parámetros genéticos (Cardellino \& Rovira, 1987).

\section{RESULTADOS Y DISCUSIÓN}

Los parámetros de calidad de agua en cuanto a temperatura tuvieron un promedio de $24,7 \pm 0,8^{\circ} \mathrm{C}$ durante el día; el $\mathrm{pH}$ estuvo entre 6 y 7,5 ; la dureza entre 17,1 y $34,2 \mathrm{mg} / \mathrm{L}$; la alcalinidad presentó $34,2 \mathrm{mg} / \mathrm{L}$ y la concentración de oxígeno disuelto estuvo mayor a $4,5 \mathrm{mg} / \mathrm{L}$. Estuvieron entre el rango de las condiciones adecuadas para cultivo de peces dulceacuícolas (Atencio, 2001).

En la tabla 1, se muestran los resultados fenotípicos de las variables de crecimiento (peso, LT, LE y espesor corporal), a lo largo del estudio. 
Tabla 1. Peso, longitud total, longitud estándar y ancho del cuerpo a lo largo del estudio en cachama blanca (Piaractus brachypomus).

\begin{tabular}{|c|c|c|c|c|c|c|c|c|}
\hline \multirow[b]{2}{*}{$\begin{array}{l}\text { Edad } \\
\text { (días) }\end{array}$} & \multicolumn{2}{|l|}{$\operatorname{Peso}(g)$} & \multicolumn{2}{|c|}{ Longitud total (mm) } & \multicolumn{2}{|c|}{ Longitud estándar (mm) } & \multicolumn{2}{|c|}{ Ancho del cuerpo (mm) } \\
\hline & $\begin{array}{c}\text { Media } \pm \text { desviación } \\
\text { estándar }\end{array}$ & $\begin{array}{l}\mathrm{CV} \\
(\%)\end{array}$ & $\begin{array}{l}\text { Media } \pm \text { des- } \\
\text { viación estándar }\end{array}$ & $\begin{array}{l}\text { CV } \\
(\%)\end{array}$ & $\begin{array}{c}\text { Media } \pm \text { desviación } \\
\text { estándar }\end{array}$ & $\begin{array}{l}\text { CV } \\
(\%)\end{array}$ & $\begin{array}{l}\text { Media } \pm \text { des- } \\
\text { viación estándar }\end{array}$ & $\mathrm{CV}(\%)$ \\
\hline 75 & $64,4 \pm 15,60$ & 24,21 & $12,57 \pm 2,47$ & 19,7 & $11,08 \pm 2,01$ & 18,19 & $1,74 \pm 0,37$ & 21,42 \\
\hline 110 & $180 \pm 29,10$ & 16,11 & $104,4 \pm 14,7$ & 14,08 & $91 \pm 21,63$ & 23,77 & $13,97 \pm 2,26$ & 16,8 \\
\hline 145 & $282,3 \pm 42,1$ & 14,92 & $208,5 \pm 14,7$ & 7,05 & $184,6 \pm 21,6$ & 11,72 & $29,21 \pm 2,26$ & 7,74 \\
\hline
\end{tabular}

Como se puede apreciar, los coeficientes de variación del peso, independientemente de la edad, fueron en todos los casos superiores en la longitud y ancho del cuerpo. Para el peso en canal y rendimiento en filete, los coeficientes de variación fueron similares a los del peso al sacrificio y longitud estándar, mientras que, para el rendimiento en canal y longitud total, fueron bajos; la EIMD, presentó el más alto coeficiente de variación con un valor de 19,15\%.
En la tabla 2, se muestran los resultados fenotípicos y los coeficientes de variación de los caracteres de calidad: humedad, ceniza, grasa y proteína de los peces a talla comercial de 180 días, en donde al igual que ocurrió con las demás variables, no se encontró un efecto significativo del estanque ( $\mathrm{p}>0,05)$. Respecto de los coeficientes de variación, la grasa mostró el valor más alto, mientras que la proteína y humedad indicaron valores menores al 6\%.

Tabla 2. Variables de crecimiento, de la canal y de calidad, al sacrificio a 180 días de edad, en cachama blanca (Piaractus brachypomus), resultados fenotípicos.

\begin{tabular}{|c|c|c|}
\hline Carácter & $\begin{array}{c}\text { media } \pm \\
\text { desviación estándar }\end{array}$ & CV (\%) \\
\hline Peso (g) & $376 \pm 39,45$ & 10,49 \\
\hline Longitud estándar (LE) (mm) & $248,95 \pm 24,27$ & 9,74 \\
\hline Longitud total (LT) (mm) & $204,30 \pm 14,89$ & 7,28 \\
\hline Ancho del cuerpo (ACPO) (mm) & $31,16 \pm 2,56$ & 8,21 \\
\hline Peso en canal (PC) (g) & $249,84 \pm 24,35$ & 9,74 \\
\hline Rendimiento en canal (RC) (\%) & $89,19 \pm 6,64$ & 7,45 \\
\hline Rendimiento en filete (RF) (\%) & $31,95 \pm 3,00$ & 9,39 \\
\hline Espinas intramusculares derechas (EIMD) & $28,86 \pm 5,52$ & 19,15 \\
\hline Humedad (HD) (\%) & $79,02 \pm 2,22$ & 2,81 \\
\hline Ceniza (\%) & $1,10 \pm 0,13$ & 12,59 \\
\hline Grasa (\%) & $7,28 \pm 1,56$ & 21,96 \\
\hline Proteína (\%) & $18,36 \pm 0,95$ & 5,02 \\
\hline
\end{tabular}

Los caracteres de calidad están cobrando cada vez más importancia en la industria de la acuicultura y, en ellos, la composición del músculo juega un papel determinante; un ejemplo es el estudio de Grigorakis et al. (2002), quienes reportan para dorada (Spaurus auratus), que los valores de grasa en la composición del músculo oscilan entre 3 y $11 \%$ y, además, mantiene una relación inversa con la humedad. En cuanto al contenido de proteína, según Cruz-Casallas et al. (2012), varía entre 17 y 21\%, dependiendo de la especie, la parte del cuerpo, la nutrición y ciclo de producción. Para el caso de la cachama blanca, los mismos autores reportan un 17,63\%, lo cual, concuerda con el 18,36\% encontrado en este estudio y, de igual manera, el porcentaje de cenizas y de humedad estuvieron acordes a los reportados por Lagoon (2009). Se comprobó que no existió efecto del estanque $(\mathrm{p}>0,05)$ en ninguno de los casos para ninguna de las características evaluadas.

Las correlaciones fenotípicas y genéticas entre los caracteres de crecimiento a las edades de 75, 110, 145 y 180 días y sus heredabilidades, se muestran en la tabla 3.

Como se observa, las heredabilidades estuvieron comprendidas entre 0,28 y 0,44, para el peso; entre 0,33 y 0,41 , para la longitud y entre 0,42 y 0,82, para el espesor corporal. Como era de esperarse en cada una de las edades, las correlaciones entre el peso y la longitud fueron cercanas a la unidad, entre 0,95 y 0,99 , para las correlaciones genéticas y entre 0,89 y 0,93 , para las correlaciones fenotípicas. Las 
Tabla 3. Heredabilidad con \pm desviación estándar en la diagonal, correlaciones genéticas con \pm desviación estándar bajo la diagonal y correlaciones fenotípicas con \pm desviación estándar sobre la diagonal, para características de crecimiento en cachama blanca (Piaractus brachypomus), a diferentes edades.

\begin{tabular}{|c|c|c|c|c|c|c|c|c|c|c|c|c|}
\hline Carácter & Peso75 $_{75}$ & $\mathbf{L E}_{75}$ & $\mathbf{L T}_{75}$ & $\mathbf{A C P O}_{75}$ & Peso110 & $\mathrm{LE}_{110}$ & $\mathbf{L} \mathbf{T}_{110}$ & $\mathbf{A C P O}_{110}$ & Peso145 & $\mathrm{LE}_{145}$ & $\mathbf{L T}_{145}$ & $\mathbf{A C P O}_{145}$ \\
\hline PesO75 $_{75}$ & $0,28 \pm 0,1$ & $0,89 \pm 0,2$ & $0,92 \pm 0,1$ & $0,75 \pm 0,1$ & $0,90 \pm 0,2$ & $0,60 \pm 0,2$ & $0,70 \pm 0,1$ & $0,59 \pm 0,2$ & $0,90 \pm 0,2$ & $0,70 \pm 0,2$ & $0,86 \pm 0,1$ & $0,76 \pm 0,2$ \\
\hline $\mathrm{LE}_{75}$ & $0,99 \pm 0,3$ & $0,33 \pm 0,1$ & $0,81 \pm 0,2$ & $0,90 \pm 0,2$ & $0,81 \pm 0,1$ & $0,78 \pm 0,1$ & $0,73 \pm 0,2$ & $0,83 \pm 0,1$ & $0,72 \pm 0,2$ & $0,77 \pm 0,1$ & $0,81 \pm 0,2$ & $0,77 \pm 0,1$ \\
\hline $\mathbf{L T}_{75}$ & $0,95 \pm 0,2$ & $0,94 \pm 0,1$ & $0,32 \pm 0,2$ & $0,72 \pm 0,3$ & $0,81 \pm 0,2$ & $0,79 \pm 0,2$ & $0,88 \pm 0,1$ & $0,77 \pm 0,1$ & $0,81 \pm 0,1$ & $0,81 \pm 0,3$ & $0,86 \pm 0,1$ & $0,72 \pm 0,2$ \\
\hline $\mathbf{A C P O}_{75}$ & $0,74 \pm 0,3$ & $0,95 \pm 0,3$ & $0,77 \pm 0,3$ & $0,42 \pm 0,1$ & $0,71 \pm 0,3$ & $0,89 \pm 0,3$ & $0,81 \pm 0,1$ & $0,90 \pm 0,2$ & $0,82 \pm 0,3$ & $0,86 \pm 0,2$ & $0,81 \pm 0,1$ & $0,90 \pm 0,3$ \\
\hline Peso110 & $0,98 \pm 0,3$ & $0,86 \pm 0,3$ & $0,83 \pm 0,2$ & $0,74 \pm 0,2$ & $0,32 \pm 0,2$ & $0,93 \pm 0,4$ & $0,84 \pm 0,1$ & $0,79 \pm 0,3$ & $0,92 \pm 0,1$ & $0,81 \pm 0,1$ & $0,72 \pm 0,3$ & $0,77 \pm 0,2$ \\
\hline $\mathrm{LE}_{110}$ & $0,64 \pm 0,4$ & $0,82 \pm 0,4$ & $0,82 \pm 0,2$ & $0,90 \pm 0,3$ & $0,97 \pm 0,2$ & $0,34 \pm 0,1$ & $0,85 \pm 0,2$ & $0,84 \pm 0,1$ & $0,81 \pm 0,2$ & $0,77 \pm 0,3$ & $0,71 \pm 0,3$ & $0,64 \pm 0,1$ \\
\hline $\mathbf{L T}_{110}$ & $0,71 \pm 0,2$ & $0,78 \pm 0,2$ & $0,92 \pm 0,3$ & $0,86 \pm 0,3$ & $0,96 \pm 0,3$ & $0,93 \pm 0,2$ & $0,37 \pm 0,1$ & $0,78 \pm 0,3$ & $0,90 \pm 0,3$ & $0,86 \pm 0,2$ & $0,90 \pm 0,2$ & $0,89 \pm 0,3$ \\
\hline $\mathrm{ACPO}_{110}$ & $0,69 \pm 0,2$ & $0,91 \pm 0,2$ & $0,79 \pm 0,3$ & $0,94 \pm 0,3$ & $0,83 \pm 0,2$ & $0,89 \pm 0,2$ & $0,84 \pm 0,2$ & $0,44 \pm 0,2$ & $0,80 \pm 0,1$ & $0,83 \pm 0,3$ & $0,91 \pm 0,2$ & $0,94 \pm 0,2$ \\
\hline Peso145 & $0,92 \pm 0,2$ & $4 \pm 0,4$ & $0,84 \pm 0,3$ & $0,78 \pm 0,3$ & $3+0,1$ & $0,84 t$ & $0,91 \pm 0,1$ & $0,82 \pm 0,3$ & $0,38 \pm 0,1$ & 0,9 & 0,9 & $0,79 \pm 0,1$ \\
\hline $\mathrm{LE}_{145}$ & $0,84 \pm 0,3$ & $0,79 \pm 0,2$ & $0,88 \pm 0,1$ & $0,91 \pm 0,2$ & $0,84 \pm 0,2$ & $0,81 \pm 0,3$ & $0,80 \pm 0,2$ & $0,86 \pm 0,2$ & $0,99 \pm 0,2$ & $0,36 \pm 0,2$ & $0,88 \pm 0,2$ & $0,71 \pm 0,2$ \\
\hline $\mathbf{L T}_{145}$ & $0,82 \pm 0,1$ & $0,84 \pm 0,3$ & $0,90 \pm 0,2$ & $0,87 \pm 0,2$ & $0,77 \pm 0,3$ & $0,76 \pm 0,4$ & $0,94 \pm 0,3$ & $0,94 \pm 0,2$ & $0,96 \pm 0,3$ & $0,91 \pm 0,2$ & $0,39 \pm 0,1$ & $0,76 \pm 0,3$ \\
\hline $\mathrm{ACPO}_{145}$ & $0,63 \pm 0,4$ & $0,89 \pm 0,2$ & $0,79 \pm 0,2$ & $0,92 \pm 0,2$ & $0,79 \pm 0,3$ & $0,71 \pm 0,3$ & $0,75 \pm 0,3$ & $0,96 \pm 0,2$ & $0,82 \pm 0,3$ & $0,74 \pm 0,2$ & $0,84 \pm 0,3$ & $0,51 \pm 0,1$ \\
\hline
\end{tabular}

Peso, longitud estándar (LE), longitud total (LT), ancho del cuerpo (ACPO)

correlaciones entre pesos y longitudes fueron altas en un rango de edades consecutivas; sin embargo, dichas estimas decrecieron en magnitud a medida que incrementó la diferencia entre las edades, siendo las correlaciones fenotípicas como promedio, inferiores a las genéticas; de manera similar, las correlaciones del ancho del cuerpo, también decrecieron con el incremento de la edad.
En la tabla 4, se muestran las heredabilidades, correlaciones fenotípicas y genéticas para los caracteres de crecimiento de la canal y de calidad, a la talla de cosecha de 180 días de edad. Respecto a las heredabilidades, las estimas estuvieron comprendidas entre 0,06 y 0,32, para las características de la canal; 0,11, para EIMD y entre 0,06 y 0,12 , para las variables de calidad.

Tabla 4. Heredabilidad con \pm desviación estándar en la diagonal, correlaciones genéticas con \pm desviación estándar bajo la diagonal y correlaciones fenotípicas con \pm desviación estándar sobre la diagonal, para características de crecimiento, de la canal y de calidad en cachama blanca (Piaractus brachypomus), a tiempo de cosecha de 180 días de edad.

\begin{tabular}{|c|c|c|c|c|c|c|c|c|c|c|c|c|}
\hline Carácter & Peso & LE & LT & ACPO & PC & RC & RF & EIMD & HD & Ceniza & Grasa & $\mathbf{P}$ \\
\hline Peso & $0,44 \pm 0,1$ & $0,81 \pm 0,2$ & $0,89 \pm 0,2$ & $0,72 \pm 0,2$ & $0,86 \pm 0,2$ & $-0,32 \pm 0,1$ & $0,62 \pm 0,1$ & $0,89 \pm 0,1$ & $0,02 \pm 0,2$ & $0,08 \pm 0,1$ & $0,28 \pm 0,2$ & $0,26 \pm 0,2$ \\
\hline LE & $0,88 \pm 0,1$ & $0,41 \pm 0,2$ & $0,91 \pm 0,1$ & $0,88 \pm 0,1$ & $0,89 \pm 0,2$ & $-0,18 \pm 0,1$ & $0,64 \pm 0,2$ & $0,82 \pm 0,0$ & $-0,12 \pm 0,1$ & $0,07 \pm 0,2$ & $0,32 \pm 0,1$ & $0,24 \pm 0,1$ \\
\hline LT & $0,91 \pm 0,1$ & $0,94 \pm 0,1$ & $0,32 \pm 0,1$ & $0,82 \pm 0,1$ & $0,81 \pm 0,1$ & $-0,27 \pm 0,0$ & $0,87 \pm 0,1$ & $0,67 \pm 0,1$ & $0,01 \pm 0,1$ & $0,07 \pm 0,1$ & $0,23 \pm 0,3$ & $0,20 \pm 0,3$ \\
\hline ACPO & $0,87 \pm 0,2$ & $0,96 \pm 0,2$ & $0,87 \pm 0,1$ & $0,82 \pm 0,1$ & $0,69 \pm 0,3$ & $-0,42 \pm 0,2$ & $0,81 \pm 0,2$ & $0,90 \pm 0,2$ & $-0,21 \pm 0,2$ & $0,06 \pm 0,2$ & $0,14 \pm 0,1$ & $0,09 \pm 0,1$ \\
\hline PC & $0,98 \pm 0,2$ & $0,86 \pm 0,2$ & $0,83 \pm 0,1$ & $0,74 \pm 0,2$ & $0,32 \pm 0,1$ & $-0,13 \pm 0,3$ & $0,66 \pm 0,1$ & $0,80 \pm 0,1$ & $0,04 \pm 0,1$ & $0,18 \pm 0,3$ & $0,32 \pm 0,2$ & $0,27 \pm 0,1$ \\
\hline RC & $-0,43 \pm 0,3$ & $-0,21 \pm 0,2$ & $-0,38 \pm 0,3$ & $-0,46 \pm 0,1$ & $-0,87 \pm 0,2$ & $0,06 \pm 0,1$ & $0,88 \pm 0,0$ & $-0,84 \pm 0,2$ & $0,11 \pm 0,2$ & $0,13 \pm 0,1$ & $0,35 \pm 0,3$ & $0,34 \pm 0,1$ \\
\hline RF & $0,63 \pm 0,2$ & $0,78 \pm 0,1$ & $0,92 \pm 0,3$ & $0,86 \pm 0,2$ & $0,71 \pm 0,2$ & $0,93 \pm 0,1$ & $0,48 \pm 0,1$ & $0,42 \pm 0,1$ & $0,13 \pm 0,1$ & $0,16 \pm 0,3$ & $0,35 \pm 0,1$ & $-0,19 \pm 0,2$ \\
\hline EIMD & $0,97 \pm 0,1$ & $0,91 \pm 0,2$ & $0,79 \pm 0,3$ & $0,94 \pm 0,2$ & $0,83 \pm 0,2$ & $-0,99 \pm 0,2$ & $0,50 \pm 0,2$ & $0,11 \pm 0,1$ & $0,01 \pm 0,2$ & $0,25 \pm 0,2$ & $0,15 \pm 0,1$ & $0,34 \pm 0,2$ \\
\hline HD & $-0,04 \pm 0,1$ & $-0,26 \pm 0,2$ & $0,04 \pm 0,1$ & $-0,26 \pm 0,2$ & $0,09 \pm 0,1$ & $-0,16 \pm 0,3$ & $0,15 \pm 0,1$ & $0,02 \pm 0,2$ & $0,09 \pm 0,1$ & $0,26 \pm 0,1$ & $-0,67 \pm 0,2$ & $-0,29 \pm 0,1$ \\
\hline Ceniza & $0,14 \pm 0,2$ & $0,09 \pm 0,1$ & $0,05 \pm 0,1$ & $0,13 \pm 0,1$ & $0,21 \pm 0,1$ & $0,17 \pm 0,2$ & $0,18 \pm 0,2$ & $0,26 \pm 0,1$ & $0,19 \pm 0,1$ & $0,12 \pm 0,1$ & $0,24 \pm 0,1$ & $0,05 \pm 0,0$ \\
\hline Grasa & $0,32 \pm 0,1$ & $0,28 \pm 0,1$ & $0,26 \pm 0,2$ & $0,17 \pm 0,1$ & $0,35 \pm 0,2$ & $0,41 \pm 0,2$ & $0,09 \pm 0,1$ & $0,24 \pm 0,2$ & $-0,78 \pm 0,2$ & $0,06 \pm 0,1$ & $0,06 \pm 0,1$ & $0,36 \pm 0,1$ \\
\hline $\mathbf{P}$ & $0,28 \pm 0,1$ & $0,36 \pm 0,1$ & $0,27 \pm 0,1$ & $0,12 \pm 0,1$ & $0,25 \pm 0,2$ & $0,39 \pm 0,2$ & $-0,26 \pm 0,1$ & $0,36 \pm 0,1$ & $-0,32 \pm 0,3$ & $0,14 \pm 0,2$ & $0,44 \pm 0,2$ & $0,08 \pm 0,0$ \\
\hline
\end{tabular}

Peso, longitud estándar (LE), longitud total (LT), ancho del cuerpo (ACPO), peso en canal (PC), rendimiento en canal (RC), rendimiento en filete $(\mathrm{RF})$, numero de espina intramuscular derecha (EIMD), humedad (HD), ceniza, grasa, proteína (P). 
Las heredabilidades, en general, para las variables de crecimiento mostraron valores de medios a altos, incluyendo el rendimiento en filete; por otra parte, el rendimiento en canal y la EIMD presentaron heredabilidades sustancialmente bajas, siendo este último carácter de gran importancia para la industria; del mismo modo, su alta y positiva correlación genética con la mayoría de características de crecimiento, sugieren que no se podría mejorar, a través de la genética cuantitativa. Las correlaciones genéticas entre RC y características de crecimiento atípicas fueron moderadamente negativas, pero cercanas a 1 con RF. A su vez, para las características de calidad y de crecimiento: peso, longitud y espesor corporal, sus correlaciones fueron sustancialmente bajas y negativas para la humedad, con la mayoría de variables. En el caso del rendimiento canal, las correlaciones genéticas, en general, fueron negativas entre todas las variables, mientras que, para el peso en canal, sus correlaciones fenotípicas y genéticas con los caracteres de crecimiento fueron altas y positivas. Por otra parte, las correlaciones genéticas y fenotípicas entre los caracteres de calidad fueron generalmente bajas y respecto a las correlaciones entre los caracteres de calidad y los de crecimiento, las fenotípicas fueron siempre más bajas que las genéticas para la mayoría de las variables.

De particular interés, el peso, característica que independientemente de la edad, mostró una heredabilidad promedio media a alta, de $0,35 \pm 0,16$, lo cual, está en magnitud similar con lo reportado por Navarro et al. (2009a), de 0,31 $\pm 0,07$, en dorada; a su vez, con los de Guan et al. (2016), de 0,34 \pm 0,11, en rodaballo y los de Whatmore et al. (2013), de 0,23 \pm 0,13, en cola amarilla (Seriola lalandi).

En el caso de la longitud, se han descrito estimas medias a altas, en carpa herbívora (Ctenopharyngodon idella), a distintas edades, entre 0,38 y 0,41, por Fu et al. (2016); en panga (Pangasianodon hypophthalmus), de 0,24 \pm 0,09, por Nguyen et al. (2012), mientras que en dorada, a 163 días, de $0,11 \pm 0,03$, por García-Celdrán et al. (2015c), valores que son coherentes con $0,35 \pm 0,17$, encontrados en el presente estudio.

Para todos los reportes de heredabilidad, anteriormente mencionados, las estimas de longitud y de peso son, en general, similares, esto supone que la pretensión de seleccionar para aumento de longitud, con el propósito de elevar indirectamente el peso, es una alternativa justificable, máxime en condiciones de campo, cuando no está disponible una balanza de precisión y se tiene un bajo coeficiente de variación; por consiguiente, es más reiterado que la variable peso, lo que indica mayores posibilidades en la intensidad de selección. Una observación similar fue hecha por Bernal \& Gallego (2016); en este sentido, Skagemo et al. (2014) han demostrado la utilidad de usar la longitud, en vez del peso, en los índices de selección genética para salmón del Atlántico, tanto en talla de comercialización como de madurez sexual.

A pesar de que las heredabilidades en este estudio estuvieron en rangos similares a los reportados para otras especies, se identificó un ligero aumento en las magnitudes a mayor edad para el caso de las variables de crecimiento, posiblemente, debido a que los componentes genéticos están en función de las frecuencias genéticas y pueden, por ello, diferir de una población a otra; es de esperar que poblaciones de censo reducido, que han perdurado durante un tiempo, lo suficientemente largo ,como para alcanzar un grado considerable de fijación, muestren heredabilidades menores, que las de censo elevado.

En cuanto a las características de la canal, la variación genética aditiva que ha sido descrita en distintas especies, abarca desde valores bajos hasta muy altos. Para el caso de peso en canal y rendimiento en canal, en dorada, García-Celdrán et al. (2015a) reportaron estimas de $0,24 \pm 0,06$ y $0,11 \pm 0,05$, mientras que en cola amarilla, Whatmore et al. (2013), obtuvieron estimas de 0,31 $\pm 0,15$ y 0,19 $\pm 0,11$, respectivamente; para este estudio, se encontraron valores de 0,32 $\pm 0,17$ y $0,06 \pm 0,04$, respectivamente. En cuanto al rendimiento en filete, los reportes indican una notable variación genética aditiva, en coherencia con Fernandes et al. (2015) que, dentro del programa de mejora de la tilapia nilótica (Oreochromis niloticus), reportaron valores de $0,20 \pm 0,05$, a los 147 días, en contraste con lo encontrado en este estudio, de $0,48 \pm 0,19$. Sin duda esto representa un buen punto de partida para su mejoramiento, teniendo en cuenta que es una característica del producto final que tiende a tener una baja variación genética.

En cuanto EIMD, en la presente población de cachama blanca representó una baja variabilidad genética aditiva, con una heredabilidad de 0,11 $\pm 0,05$, demostrando que la pretensión de mejorar esta característica va a depender de técnicas genéticas moleculares, pero no a través de la genética cuantitativa, máxime con la alta correlación genética y fenotípica, que presentó con la mayoría de características de crecimiento.

Respecto a las correlaciones genéticas entre edades es interesante estimarlas, en el sentido de que permiten tomar decisiones para preseleccionar a talla temprana, dependiendo de la magnitud de las mismas. En general, como ha sido descrito en diversas especies, estas correlaciones son bajas entre edades distantes; esto concuerda con las correlaciones genéticas encontradas en este estudio, entre 75 y 110 días de edad, respecto con la edad del sacrificio, sugiriendo que no sería apropiada una selección en fases tempranas del desarrollo de cachama blanca. Por el contrario, las correlaciones genéticas entre edades cercanas al sacrificio suelen ser altas, entre 0,60 a 0,80 , aproximadamente, según Charo-Karisa et al. (2007). Lo anterior coincide con las correlaciones genéticas reportadas en el presente estudio en 145 días y la talla de sacrificio entre pesos y longitudes. Del mismo modo, las correlaciones genéticas entre espesor corporal, aunque son más altas entre edades consecutivas, son superiores que entre edades distantes, al igual que se ha encontrado en otras estudios, según Maluwa et al. (2006).

Los caracteres de la canal, actualmente, se destacan frente a otras características en la comercialización, como pez entero. En este sentido, el conocimiento de las correlaciones genéticas entre caracteres de crecimiento y de la canal es de gran importancia a la hora de tomar decisiones en los procesos de selección genética; de acuerdo con Haffray et al. (2014), en el caso del rendimiento de la canal y en filete, sus estimas frente al peso y la longitud son consistentes entre especies, siendo muy altas y positivas, como ha sido descrito en el presente estudio. Estos resultados ponen de manifiesto que, 
en cachama blanca, el rendimiento del filete aumenta a medida que lo hace el peso o la longitud de los peces, tanto desde el punto de vista fenotípico como genético, por las altas y positivas correlaciones. Entonces, inicialmente, parece adecuado, que la mejora de los caracteres de la canal en cachama blanca queda a expensas de la selección directa para la longitud, teniendo en cuenta que la evaluación de estos caracteres presenta la desventaja de ser laboriosa y requiere de tecnificación, a la vez que del sacrificio de los peces.

Con relación a los caracteres de calidad en peces, han sido descritos valores de heredabilidades muy diversos. De los caracteres de la composición de la carne, el más estudiado ha sido la grasa muscular, del cual, se han descrito valores altos de 0,47 , según Gjerde \& Gjedrem (1984) y bajos, de $0,04 \pm 0,08$, según Quinton et al. (2005), lo que concuerda con las estimas del presente estudio, de $0,06 \pm 0,15$. Por su parte, García-Celdrán et al. (2015b), en un estudio en dorada, reporta una heredabilidad media de $0,20 \pm 0,06$.

En el caso del porcentaje de humedad, las estimas de heredabilidad son también heterogéneas en las distintas especies en el que ha sido descrito, en dorada; Navarro et al. (2009b) describieron valores bajos, tanto para la humedad de $0,09 \pm 0,03$ como para la ceniza, de $0,08 \pm 0,03$, lo que concuerda con los valores encontrados en este trabajo, de $0,09 \pm 0,18$ y $0,12 \pm 0,13$, respectivamente. Por otra parte, las relaciones genéticas entre las características, en general, fueron bajas, como se ha descrito para especies, como el panga, donde Nguyen et al. (2012) destacan una correlación genética de 0,35 entre características de la canal y de crecimiento, que favorece indirectamente, a través de una mejora del crecimiento, el porcentaje de grasa muscular, mejorando la calidad de la carne.

Finalmente, señalar que querer mejorar el crecimiento en cachama blanca por medio de la longitud estándar en vez del peso y la longitud total es un criterio adecuado, puesto que presenta una heredabilidad más alta que la longitud total y, a su vez, es de más fácil medición y más repetible respecto del peso. Adicionalmente, ambas características presentan heredabilidades similares y altas correlaciones genéticas en todas las edades, pero la selección directa de la longitud estándar, mejoraría indirectamente el peso de la canal, el rendimiento en filete y los caracteres de composición de la carne, especialmente, el contenido de proteína y, en menor medida, el porcentaje de cenizas, sin tener que sacrificar los peces, sin efecto sobre la morfología. Por otro lado, la correlación genética negativa entre la longitud estándar y el rendimiento en canal, sugiere la inclusión de éste en los índices de selección.

A manera de colofón, todos estos resultados revelan el potencial para mejorar algunas características de crecimiento y de carcasa, a través de la explotación de la variación genética aditiva, que constituyen, en sí mismos, transferencia de conocimiento, puesto que han sido realizados con la genética y animales propios de empresas del sector, demostrando que es posible implementar un programa de mejora genética, conjugando a temprana edad el sistema de marcaje físico, implante de elastómero fijo (Bernal-Buitrago et al. 2017), sin dejar de lado la idiosincrasia de la industria.
Cabe resaltar que, queda en evidencia, el requerimiento de futuras investigaciones genéticas, no solo cuantitativas, sino también moleculares, que permitan seguir avanzando en el conocimiento del comportamiento de la espina intramuscular, en pro del mejoramiento de la especie, para así buscar estrategias que conlleven a su disminución, puesto que la pretensión de mejorar esta característica, va a depender de técnicas genéticas moleculares, pero no a través de la genética cuantitativa, máxime con la alta correlación genética y fenotípica, que presentó con las características de crecimiento.

Conflicto de interés: El manuscrito fue preparado y revisado con la participación de todos los autores, quienes declaramos que no existe conflicto de interés que ponga en riesgo la validez de los resultados presentados. Financiación: Este estudio fue financiado por la Dirección General de Investigaciones (DGI) de la Universidad de los Llanos y el programa de Jóvenes Investigadores de Colciencias.

\section{REFERENCIAS}

1. ATENCIO, G. 2001. Producción de alevinos de especies nativas. Rev. MVZ Córdoba. 6(1):9-14.

2. AUTORIDAD NACIONAL DE ACUICULTURA Y PESCA-AUNAP; ORGANIZACIÓN DE LAS NACIONES UNIDAS PARA LA ALIMENTACIÓN Y LA AGRICULTURA-FAO; MINISTERIO DE AGRICULTURA Y DESARROLLO RURAL-MADR. 2014. Plan Nacional para el Desarrollo de la Acuicultura Sostenible en Colombia PlaNDAS. Bogotá, Colombia. 84p.

3. BERNAL-BUITRAGO, G.; VALDERRAMA-DIAZ, J.; SANTOS-SALAMANCA, J.; RIAÑO-GÓMEZ, A.; CRUZ-CASALLAS, P.; MEDINA-ROBLES, V. 2017. Evaluación del sistema de marcaje físico visible (V.I.E) en cachama blanca (Piaractus brachypomus). Orinoquia. 20(2):256-260.

4. BERNAL, F.; GALLEGO, F. 2016. Estimación de parámetros genéticos para peso y talla a diferentes edades en yamú (Brycon amazonicus). Rev. U.D.C.A Act. \& Div. Cient. 19(1):123130. https://doi.org/10.31910/rudca.v19.n1.2016.117

5. CAMPOS, J. 2004. Melhoramento genético aplicado à produção animal. Ed. FEPMVZ (Belo Horizonte). 607p.

6. CARDELLINO, R.; ROVIRA, J. 1987. Mejoramiento Genetico Animal Ed. Agropecuária Hemisfério Sur (Uruguay, Montevideo). 253p.

7. CHARO-KARISA, H.; BOVENHUIS, H.; REZK, M.; PONZONI, R.; VAN ARENDONK, J.; KOMEN, H. 2007. Phenotypic and genetic parameters for body measurements, reproductive traits and gut length of Nile tilapia (Oreochromis niloticus) selected for growth in low-input earthen ponds. Aquaculture. 273:15-23. https://doi.org/10.1016/ j.aquaculture.2007.09.011 
8. CRUZ-CASALLAS, N.; CRUZ-CASALLAS, P.; SUÁREZ-MAHECHA, H. 2012. Characterization of the nutritional quality of the meat in some species of catfish: a review. Rev. Facultad Nacional de Agronomía, Medellín. 65(2):67996809.

9. FERNANDES, A.; DE ALMEIDA SILVA, M.; DE ALVARENGA, E.; DE ALENCAR TEIXEIRA, E.; DA SILVA JUNIOR, A.; DE OLIVEIRA ALVES, G.; DE SALLES, S.; MANDUCA, L.; TURRA, E. 2015. Morphometric traits as selection criteria for carcass yield and body weight in Nile tilapia (Oreochromis niloticus L.) at five ages. Aquaculture. 446:303-309. https://doi.org/10.1016/j.aquaculture.2015.05.009

10. FU, J.; SHEN, Y.; XU, X.; LI, J. 2016. Genetic parameter estimates for growth of grass carp, Ctenopharyngodon idella, at 10 and 18 months of age. Aquaculture. 450:342-348. https:/ / doi.org/10.1016/j.aquaculture.2015.08.018

11. GARCÍA-CELDRÁN, M.; RAMIS, G.; MANCHADO, M.; ESTÉVEZ, A.; AFONSO, J.; ARMERO, E. 2015a. Estimates of heritabilities and genetic correlations of carcass quality traits in a reared gilthead sea bream (Sparus aurata L.) population sourced from three broodstocks along the Spanish coasts. Aquaculture. 446:175-180. https://doi. org/10.1016/j.aquaculture.2015.04.028

12. GARCÍA-CELDRÁN, M.; RAMIS, G.; MANCHADO, M.; ESTÉVEZ, A.; NAVARRO, A.; ARMERO, E. 2015b. Estimates of heritabilities and genetic correlations of raw flesh quality traits in a reared gilthead sea bream (Sparus aurata L.) population sourced from broodstocks along the Spanish coasts. Aquaculture. 446:181-186. https://doi. org/10.1016/j.aquaculture.2015.04.030

13. GARCÍA-CELDRÁN, M.; RAMIS, G.; MANCHADO, M.; ESTÉVEZ, A.; AFONSO, J.; MARÍA-DOLORES, E.; PEÑALVER, J.; ARMERO, E. 2015c. Estimates of heritabilities and genetic correlations of growth and external skeletal deformities at different ages in a reared gilthead sea bream (Sparus aurata L.) population sourced from three broodstocks along the Spanish coasts. Aquaculture. 445:3341. https://doi.org/10.1016/j.aquaculture.2015.04.006

14. GJEDREM, T.; ROBINSON, N.; RYE, M. 2012. The importance of selective breeding in aquaculture to meet future demands for animal protein: A review. Aquaculture. 350-353(0):117-129. https://doi.org/10.1016/j.aquaculture.2012.04.008

15. GJERDE, B.; GJEDREM, T. 1984. Estimates of phenotypic and genetic parameters for carcass traits in Atlantic salmon and rainbow trout. Aquaculture. 36(1-2):97-110. https:/ / doi.org/10.1016/0044-8486(84)90057-7
16. GRIGORAKIS, K.; ALEXIS, M.; TAYLOR, K.; HOLE, M. 2002. Comparison of wild and cultured gilthead sea bream (Sparus aurata); composition, appearance and seasonal variations. Internal J. Food Science \& Technology. 37(5):477484. https://doi.org/10.1046/j.1365-2621.2002.00604.x

17. GUAN, J.; HU, Y.; WANG, M.; WANG, W.; KONG, J.; LUAN, S. 2016. Estimating genetic parameters and genotype-byenvironment interactions in body traits of turbot in two different rearing environments. Aquaculture. 450:321-327. https://doi.org/10.1016/j.aquaculture.2015.08.014

18. HAFFRAY, P.; BUGEON, J.; RIVARD, Q.; QUITTET, B.; PUYO, S.; ALLAMELOU, J.M.; VANDEPUTTE, M.; DUPONT-NIVET, M. 2014. Reprint of: Genetic parameters of in-vivo prediction of carcass, head and fillet yields by internal ultrasound and 2D external imagery in large rainbow trout (Oncorbynchus mykiss). Aquaculture. 420-421(Supplement 1):S134-S142. https://doi.org/10.1016/j.aquaculture.2014.01.016

19. LAGOON, O. 2009. Contenido de grasa y proteina en Pygocentrus cariba, Prochilodus mariae, Plagioscion squamosissimus, Piaractus brachypomus e Hypostomus plecostomus en una laguna de inundación del Orinoco medio. Rev Científica (Maracaibo). 19(1):15-21.

20. MALUWA, A.; GJERDE, B.; PONZONI, R. 2006. Genetic parameters and genotype by environment interaction for body weight of Oreochromis shiranus. Aquaculture. 259(1-4):47-55. https://doi.org/10.1016/j.aquaculture.2006.06.033

21. MESA-GRANDA, M.; BOTERO-AGUIRRE, M. 2007. La cachama blanca (Piaractus brachypomus), una especie potencial para el mejoramiento genético. Rev. Col. Ciencias Pecuarias. 20(1):79-86.

22. NAVARRO, A.; ZAMORANO, M.; HILDEBRANDT, S.; GINÉS, R.; AGUILERA, C.; AFONSO, J. 2009a. Estimates of heritabilities and genetic correlations for growth and carcass traits in gilthead seabream (Sparus auratus L.), under industrial conditions. Aquaculture. 289(3-4):225-230. https://doi.org/10.1016/j.aquaculture.2008.12.024

23. NAVARRO, A.; ZAMORANO, M.; HILDEBRANDT, S.; GINÉS, R.; AGUILERA, C.; AFONSO, J. 2009b. Estimates of heritabilities and genetic correlations for body composition traits and $G \times E$ interactions, in gilthead seabream (Sparus auratus L.). Aquaculture. 295(3-4):183-187. https:/ / doi.org/10.1016/j.aquaculture.2009.07.012

24. NGUYEN, V.; KLEMETSDAL, G.; ØDEGÅRD, J.; GJØEN, H. 2012. Genetic parameters of economically important traits recorded at a given age in striped catfish (Pangasianodon bypophthalmus). Aquaculture. 344-349(0):82-89. https://doi. org/10.1016/j.aquaculture.2012.03.013 
25. ORGANIZACIÓN DE LAS NACIONES UNIDAS PARA LA ALIMENTACIÓN Y LA AGRICULTURA-FAO. 2016. El Estado Mundial de la Pesca y la Acuicultura. Roma. 224p.

26. QUINTON, C.; MCMILLAN, I.; GLEBE, B. 2005. Development of an Atlantic salmon (Salmo salar) genetic improvement program: genetic parameters of harvest body weight and carcass quality traits estimated with animal models. Aquaculture. 247(1-4):211-217. https://doi.org/10.1016/ j.aquaculture.2005.02.030
27. SKAGEMO, V.; SONESSON, A.; MEUWISSEN, T.; LILLEHAMMER, M.; RYE, M. 2014. The dissemination of genetic improvement in salmon production. Aquaculture. 422-423(0):78-83. https://doi.org/10.1016/j.aquaculture.2013.11.028

28. WHATMORE, P.; NGUYEN, N.H.; MILLER, A.; LAMONT, R.; POWEL, D.; D’ANTIGNANA, T.; BUBNER, E.; ELIZUR, A.; KNIBB, W. 2013. Genetic parameters for economically important traits in yellowtail kingfish Seriola lalandi. Aquaculture. 400-401:77-84. https://doi.org/10.1016/ j.aquaculture.2013.03.002 